\title{
Крайнова О.С.
}

Кандидат экономических наук, доцент кафедры товароведения и экспертизы качества,

Институт пищевых технологий и дизайна, филиал ГБОУ ВО «Нижегородский государственный инженерно-экономический университет»

\section{ОПТИМИЗАЦИЯ ЛОГИСТИЧЕСКОЙ ПОДСИСТЕМЫ СКЛАДИРОВАНИЯ: ПРАКТИКА ВНЕДРЕНИЯ WМS}

\begin{abstract}
Аннотация
В статье представлены результаты авторского исследования систем управления складом класса WMS. Выявлены основные и дополнительные преимущества внедрения автоматизированных систем управления складом, представлен обзор эффектов от внедрения на примере оптово-розничного предприятия. Показана эффективность управления логистическими потоками в подсистеме складирования за счет применения WMS.
\end{abstract}

Ключевые слова: система управления складом (WMS), автоматизация складских процессов, оптимизация логистических потоков

Keywords: Warehouse Management System (WMS), automated warehouse processes , optimization of logistics flows.

На сегодняшний день не вызывает сомнения утверждение о том, что автоматизированная складская логистика позволяет координировать все складские операции, поддерживает бесперебойную работу, задает алгоритмы хранения и обработки грузов, физического распределения между заказчиками, а грамотно выстроенная логистика склада и применение новых технологий - необходимое условие для успешного развития и повышения конкурентоспособности компании. 
Поскольку для любого предприятия склад - это источник постоянных затрат, то внедрение новых сервисов и автоматизация складских процессов позволяют предприятию оптимизировать затраты на содержание склада и повысить его эффективность.

В представленных материалах авторского исследования приводятся материалы оптово-розничного предприятия, основным видом деятельности которого является дистрибуция алкогольных и пивных, безалкогольных напитков, табачных изделий, а также товаров «у кассы» (снэки, жевательная резинка и пр.) в Нижегородской области.

Наиболее характерные проблемы склада предприятия, выявленные в ходе исследования, - это пересортица, низкая скорость комплектации заказа, ошибки комплектации и подбора заказов, сложность проведения ревизии, недостачи и длительная по времени инвентаризация.

Поскольку операционная деятельность предприятия связана с большим массивом отчетной документации, умалять значение каждой бумаги, конечно же, не имеет смысла. Все они призваны упорядочить и систематизировать деятельность компании. Тем не менее, несмотря на такое важное назначение, невозможно оставить в стороне минусы ведения подобной отчетности в формате «бумажной» технологии. Первый и основной недостаток документации - большое её количество, которое значительно тормозит темпы работы и увеличивает временные затраты на складской учет. Это очевидно и бесспорно. Значения, указанные в номенклатурах и накладных должны тщательно проверяться во избежание ошибок и просчетов. Такой контроль делает складской учет более растянутым по времени. Конечно, подобная ситуация так же в некоторой мере связана и с ритмом деятельности персонала. Именно поэтому система управления складом должна учитывать и человеческий фактор.

Для дальнейшего предупреждения выявленных проблем на складах предприятия было принято решение о внедрении программы HOST WMS и 
перестройки складов под эту программу, используя первоначально сравнительный анализ предлагаемых на рынке WMS систем [1-3].

Системы класса WMS (Warehouse Management System) - это системы управления складом, позволяющие выстраивать операционную работу на складе автоматически на основе введения адресации на складе. Другими словами, как минимум склад делится на некоторые зоны для облегчения поиска того или иного товара, как максимум - каждому паллетоместу присваивается свой код, и это место уже называется «ячейкой», затем ячейки объединяются в зоны, в результате чего формируется карта склада с адресным хранением.

Технология выполнения операций с грузом, таки образом, позволяет проводить все действия внутри склада с указанием определенной ячейки. $\mathrm{K}$ примеру, приняли товар от поставщика - указали в качестве ячейки приемки зоны у ворот, разместили паллету с товаром в стеллаж - указали, что товар переместился из зоны приемки в ячейку хранения, отобрали товар для клиента - зафиксировали перемещение из ячейки отбора в зону отгрузки и т.д.

Все эти перемещения можно учитывать и с помощью бумажных документов - на каждую операцию печатается нужный документ и выдается исполнителю. Или наоборот - исполнитель подходит в диспетчерскую, сканирует свой бейдж (с имеющимся ID) и из принтера автоматически распечатывается очередное задание для выполнения.

У этого метода много плюсов и минусов, но он остается самым простым и дешевым, т.к. кроме самого программы WMS и компьютера, на который ПО будет установлено (новый сервер или уже имеющийся компьютер), а также принтера - больше ничего не нужно. Предвосхищая уточнения экспертов о процедуре внедрения системы с позиции подготовки мест хранения, отметим, что в состав работ по автоматизации склада и его подготовке войдут сопровождение стеллажей, поддонов и пр. этикетками (как правило, с 
нанесением штрих-кодов), занесение информации в систему и обучение пользователей работе с новой системой.

Но если есть желание еще больше повысить отдачу от используемой WMS системы, то следует воспользоваться технологией, когда вместо бумаги каждому работнику выдается маленький персональный компьютер - терминал сбора данных (в специализированных отраслевых изданиях можно встретить понятие DOS-терминала). Он соединятся с основным компьютером, используя технологию передачи данных «Wi-Fi», и отображает на своем экране задания для пользователя, а пользователь, в свою очередь, фиксирует факт выполнения задания в режиме реального времени.

Для повышения скорости работы с терминалом и снижению количества ошибок на складе вводится автоматическая идентификация с помощью штрихкодирования, причем наносятся штрих-коды как на товар, так и паллету, этикетку ячеек. На терминале штрих-код считывается с помощью лазера одним нажатием кнопки и сотрудник быстро и точно подтверждает свои действия относительно выбранного товара, отправной точки его хранения на конкретном адресе и конечной точки комплектации и/или отбора.

Безусловно, внедрение WMS системы требует и дополнительных аппаратных средств, к которым относятся терминалы сбора данных, беспроводное оборудование и принтеры штрих-кодов. В свою очередь, это увеличивает стоимость проекта, но позволяет использовать технологии, значительно повышающие скорость выполнения работ, точность складских остатков и в конечном счете удовлетворенность клиентов при снижении затрат на склад. Так, состав типового обеспечения внедрения продукта WMS выглядит следующим образом:

1. программа, которая осуществляет управление складом и может использовать все вышеописанные возможности;

2. компьютер (или сервер), где будет работать эта программа; 
3. компьютер оператора и/или начальника склада, который будет работать с программой в режиме рабочий станции;

4. принтер формата А4 для печати документов и штрих-кодов;

5. мобильные беспроводные компьютеры с встроенным сканером штрихкодов (терминалы сбора данных, DOS-терминалы);

6. беспроводная сеть для обеспечения работы терминалов сбора данных;

7. услуги по развертыванию системы на складе.

Для предприятия - объекта авторского исследования, была предложена программа по автоматизации склада HOST WMS. Она соответствует всем вышеперечисленным параметрам WMS системы и специально «наполнена» опциями для данного предприятия.

По результатам внедрения проекта по автоматизации склада HOST WMS складской учет изменяет как своё качество, так и оперативность: с помощью системы можно отследить остатки товара на складе и получить отчеты по остаткам на любую дату.

Преимущества автоматизированного складского учета в рамках проекта были заявлены следующим образом: повышение скорости работы, снижение роли человеческого фактора, сокращение ошибок, контроль остатков на складе и новых поступлений, эффективное использование складских площадей и снижение издержек. Немаловажным доводом в пользу применения системы являлось и то, что автоматизация минимизирует влияние человеческого фактора на складские процессы, решает проблему наиболее распространенных ошибок.

Как было озвучено ранее, WMS склад управляется HOT WMS, которая представляет собой сложный программно-аппаратный комплекс, в котором все складские операции, планирование и управление осуществляются в режиме реального времени. Таким образом, WMS склад базируется на технологиях адресного хранения товаров, автоматической идентификации мест хранения товара (с помощью штрихкодирования или радиочастотных меток), ав- 
томатической идентификации складской техники и персонала (штрих-код или радиочастотная метка), дистанционном управлении исполнением складских операций (с помощью мобильных промышленных компьютеров), удаленном управлении персоналом.

Внедрение WMS системы позволяет оптимизировать функции оперативного управления складом. Работники WMS склада получают прямые визуальные или голосовые указания, выполнение заданий и другие действия подтверждаются сканированием идентификационных меток товаров и мест хранения. В случае ошибки исполнителя WMS автоматически корректирует задание. Беспроводные технологии передачи данных и штрихкодирование обеспечивают высокую эффективность автоматизированного склада.

Основные преимущества формирования WMS склада заключаются в высокой скорости и точности операций, реализованной функции координирования всех служб, рациональном использовании сотрудников и техники, бесперебойной работе, сокращении финансовых потерь, возможности внесения корректив и исправления ошибок, увеличении потенциала склада.

Внедрение HOST WMS обладает и рядом дополнительных достоинств: позволяет вести мониторинг работы склада, в режиме реального времени поставляет текущую информацию, анализирует эффективность работы сотрудников, формирует отчеты. Управление складом на базе WMS системы обеспечивает эффективное использование складских площадей, оптимизацию маршрутов погрузочно-разгрузочного транспорта, рациональное распределение заданий между сотрудниками. Система позволяет максимально увеличить эффективность использования всех складских ресурсов: площадей, техники, сотрудников склада.

Складская система WMS существенно сокращает издержки, при этом возможности склада расширяются, а грузооборот увеличивается. К дополнительным преимуществам складской системы относится прозрачность, возможность объективно оценивать работу сотрудников, получать отчеты в ре- 
жиме реального времени. Внедрение на складе систем WMS класса позволяет также в режиме реального времени управлять всеми складскими операциями, оптимизируя время на их выполнение.

Система управления, основанная на адресном хранении и технологии штрихкодирования, позволяет поддерживать оптимальный порядок хранения товара, избавляет от ошибок и простоев. Автоматизированный склад может функционировать без перерыва, что существенно сокращает издержки предприятия.

Объективность заявленных эффектов от автоматизации склада естественным образом будет выявлена в течение определенного времени ее работы, однако, уже сегодня можно констатировать, что достижение суммарного экономического эффекта возможно лишь при внедрении комплексного логистического подхода к управлению потоками предприятия в рамках микрологистической системы, охватывая не только область автоматизации подсистемы складирования, но и всех функциональных подсистем.

\section{Список литературы}

1. Обзор продуктов WMS Manhattan SCALE [Электронный ресурс] // Официальный сайт компании AXELOT, эксперта в области разработки, внедрения и сопровождения ИТ-систем. 2015. http://www.axelot.ru/products/mans/ (Дата обращения 17.10.2015).

2. Обзор продуктов WMS ГК «Систематика» [Электронный ресурс] // Официальный сайт компании «TOPS Consulting», объединяющей лидирующие практики «ГК Систематика» по разработке и внедрению ИТ-бизнесрешений. 2015. URL: www.tops.ru (Дата обращения 18.10.2015).

3. Обзор продуктов Кронос: WMS на базе 1C [Электронный ресурс] // Официальный сайт компании "Белый Конь", представляющей программный продукт Кронос: WMS. 2015. URL: http://1ccronoswms.ru/cronoswms.html (Дата обращения 18.10.2015). 